\title{
Using Computer Based Simulation to Improve Learning Experience in Me- chanical Behavior of Materials Class
}

\section{Dr. Jing Zhang, Indiana University Purdue University - Indianapolis}

Dr. Jing Zhang's research interests are broadly centered on understanding the processing-structureproperty relationships in advanced ceramics and metals for optimal performance in application, and identifying desirable processing routes for its manufacture. To this end, the research group employs a blend of experimental, theoretical, and numerical approaches, focusing on several areas, including:

1. Processing-Microstructure-Property-Performance Relationships: thermal barrier coating, solid oxide fuel cell, hydrogen transport membrane, lithium-ion battery 2. Physics-based Multi-scale Models: ab initio, molecular dynamics (MD), discrete element models (DEM), finite element models (FEM) 3. Coupled Phenomena: diffusion-thermomechanical properties 4. Additve Manufacturing (AM) or 3D Printing: AM materials characterization, AM process (laser metal powder bed fusion, ceramic slurry extrusion) design and modeling

(http://www.engr.iupui.edu/ jz29/)

\section{Mr. Tejesh Charles Dube, Indiana University Purdue University, Indianapolis}

I am a Mechanical Engineering graduate student interested in structural and material science application in the field of mechanical engineering.

\section{Mr. Michael Golub, IUPUI}

Michael Golub is the Academic Laboratory Supervisor for the Mechanical Engineering department at IUPUI. He is an associate faculty at the same school, and has taught at several other colleges. He has conducted research related to Arctic Electric Vehicles and 3D printed plastics and metals. He participated and advised several student academic competition teams for several years. His team won 1st place in the 2012 SAE Clean Snowmobile Challenge. He has two masters degrees: one M.S. in Mechanical Engineering and an M.F.A. in Television Production. He also has three B.S. degrees in Liberal Arts, Mechanical Engineering, and Sustainable Energy. 


\title{
Using Computer Based Simulation to Improve Learning Experience in Mechanical Behavior of Materials Class
}

\begin{abstract}
Traditionally, the study of Mechanical Behavior of Materials class relies on solving analytical solution using a piece of paper and pen. With the advance of computer simulations, both hardware and software, there is a need to advance the education with adoption of advanced computer technologies. In this project, we recently developed a new project-based finite element modeling module in Mechanical Behavior of Materials class. Specifically, students are required to identify a project which requires to using a commercial finite element software package to simulate the mechanical response of the system. Using an example of simulating fatigue property of a component, it demonstrates that the revised engineering course becomes increasingly popular with continued growth of interests.
\end{abstract}

\section{Introduction}

Mechanical Behavior of Materials is a graduate level course offered in our engineering department. It teaches how loading and environmental conditions can influence the behavior of materials in service. It covers elastic and plastic behavior, fracture, fatigue, low- and high-temperature behavior. It also emphasizes on methods of treating these conditions in design.

Traditionally, the study of Mechanical Behavior of Materials class relies on solving analytical solution using a piece of paper and pen. Through adopting advanced FEA (finite element analysis) programs, the students have the opportunity to work on real-world issues, which is almost impossible in the traditional teaching style, which only covers examples with simple geometry and boundary conditions. With the advance of computer simulations, both hardware and software, there is a need to advance the education with adoption of advanced computer technologies.

There are some previous studies on modeling of the fatigue responses of components. For example, Emgel et al. [1] used finite element analysis to determine the various stresses in their model, then simply use hand calculated stresses to calculate expected fatigue life. Afolabi et al. [2. noted that stress concentration was a factor to consider a spinning shaft. However, they make no mention in their analysis of attempting to make slight modifications of the part to mitigate this factor. Ma $e t$ al. [3] applied to the performance analysis stage of a body frame product. Zaharia et al. [4] tested the Monte Carlo method in the Weibull software, alongside using ANSYS for fatigue life analysis. Swamy et al. observed that variation in grid size leaded to significant variation in the life of the dental implant [5], making them the first to actually test several variations within an FEA software (CATIA, in this case) over the course of a single paper. Despite this step in the right direction, they were testing the effect of mesh density rather than actively changing their design. Vinod et al. [6] tested the fatigue life of railway bar and train wheel in contact in ANSYS. Lu et al evaluated a design to ensure the life met their design target. 


\section{Objective}

In this project, the objective is to develop a project-based finite element modeling module in the Mechanical Behavior of Materials class. Specifically, students are required to identify a project which requires to using a commercial finite element software package to simulate the mechanical response of the system.

As a case study, the fatigue life of a component using finite element analysis is demonstrated to reduce the stress concentration caused by edges. The project will demonstrate the effectiveness of making small changes in geometry, while drastically changing the stress severity. In the following, it shows how the model is set up and the analysis of fatigue level. Additionally the course evaluation and assessment data are presented.

\section{Approach}

This section describes an approach to analyze and design around stress concentrations in a mechanical component as shown in Figure 1. The materials properties are summarized in Table 1 .

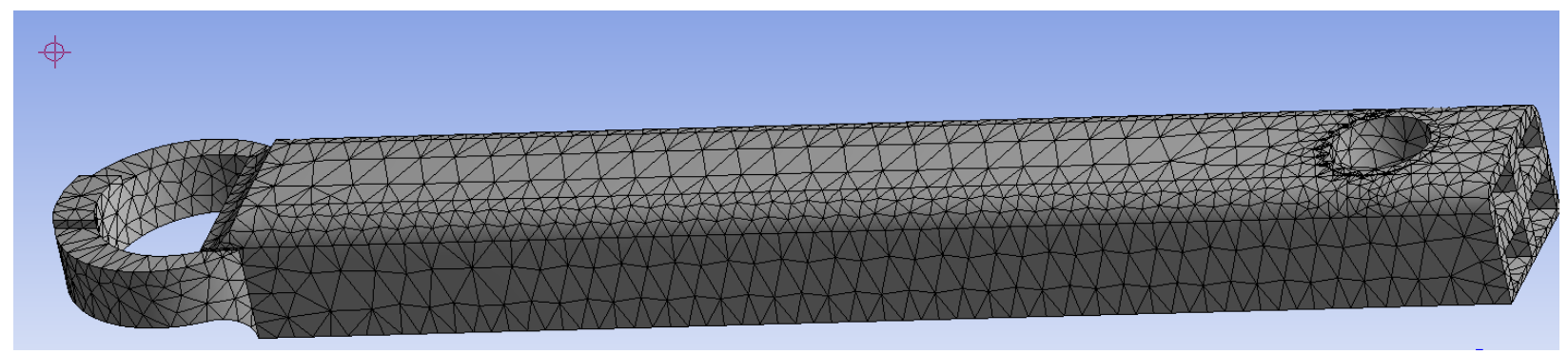

Figure1: Finite element mesh of the model

Table 1: Materials properties used in the simulation 


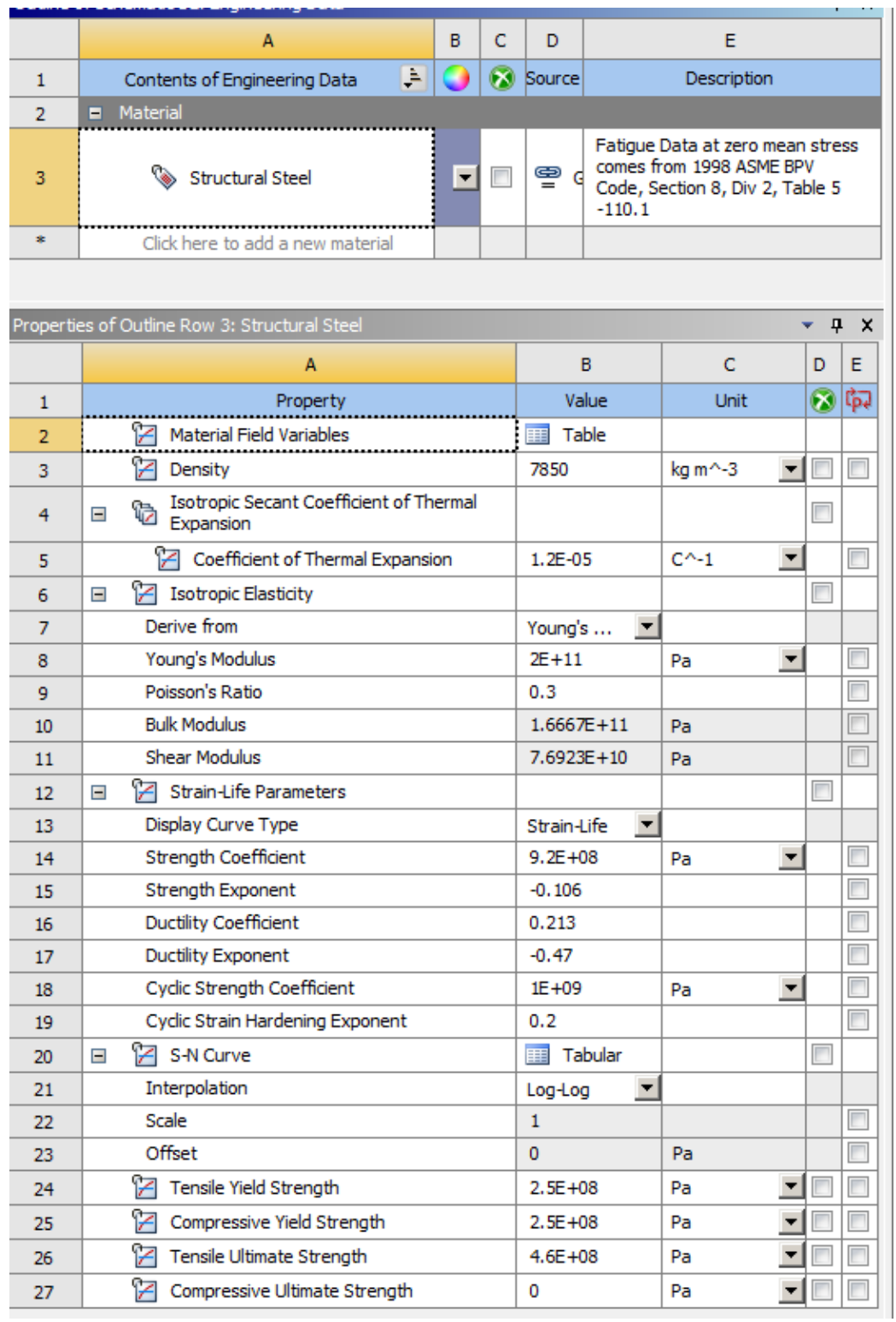

A static mechanical loading analysis is conducted first. The stress distribution is shown in Figure 2, which highlights the areas where the part would fail first. 


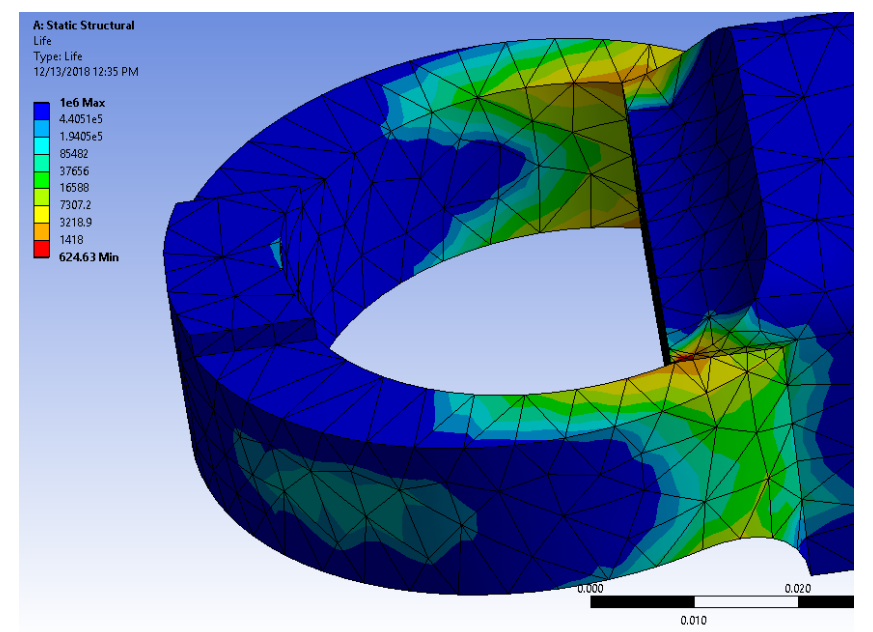

Figure 2: Simulated von Mises distribution in the static mechanical loading analysis

To improve the mechanical strength, especially the fatigue life, two different geometric designs are proposed, as shown in Figure 3 and Figure 4. As shown in the figures, some slight modifications to the left end of the model were made.

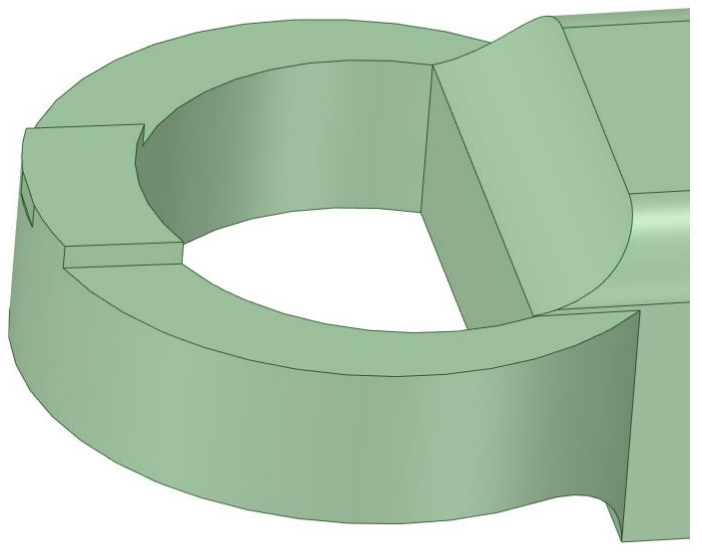

Figure 3: Design model A 


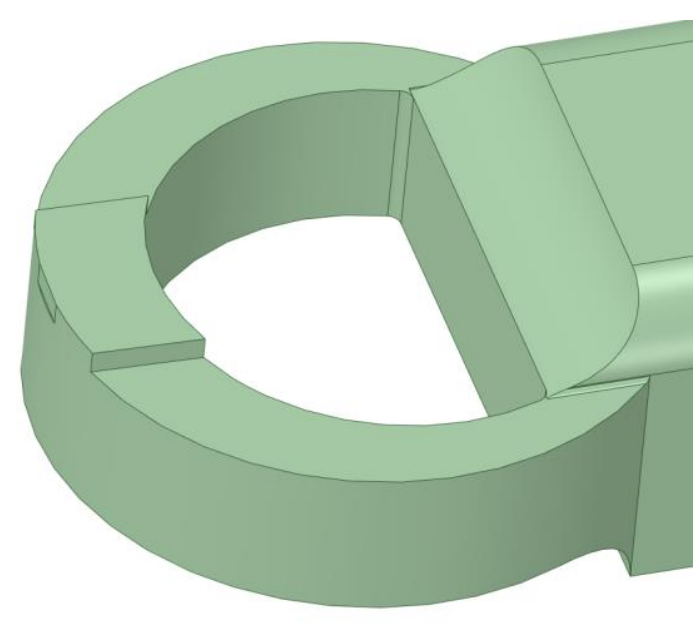

Figure 4: Design model B

The part in Figure 3, Model A, is the base model. The model in Figure 4 Model B, has had rounds with a diameter of $0.15 \mathrm{~mm}$ applied to four small edges.

The fatigue life analysis is done with the Goodman equation, using adjusted stress to account for the mean stress being equal to half of max stress. The fatigue analysis results are in Figure 5 below.

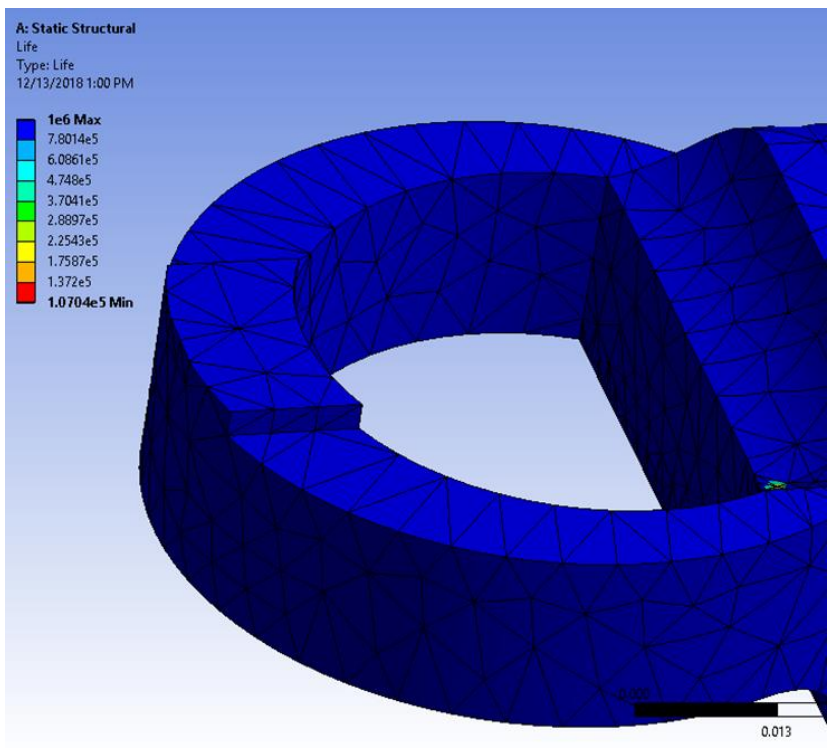

Figure 5: Fatigue life of Model A 


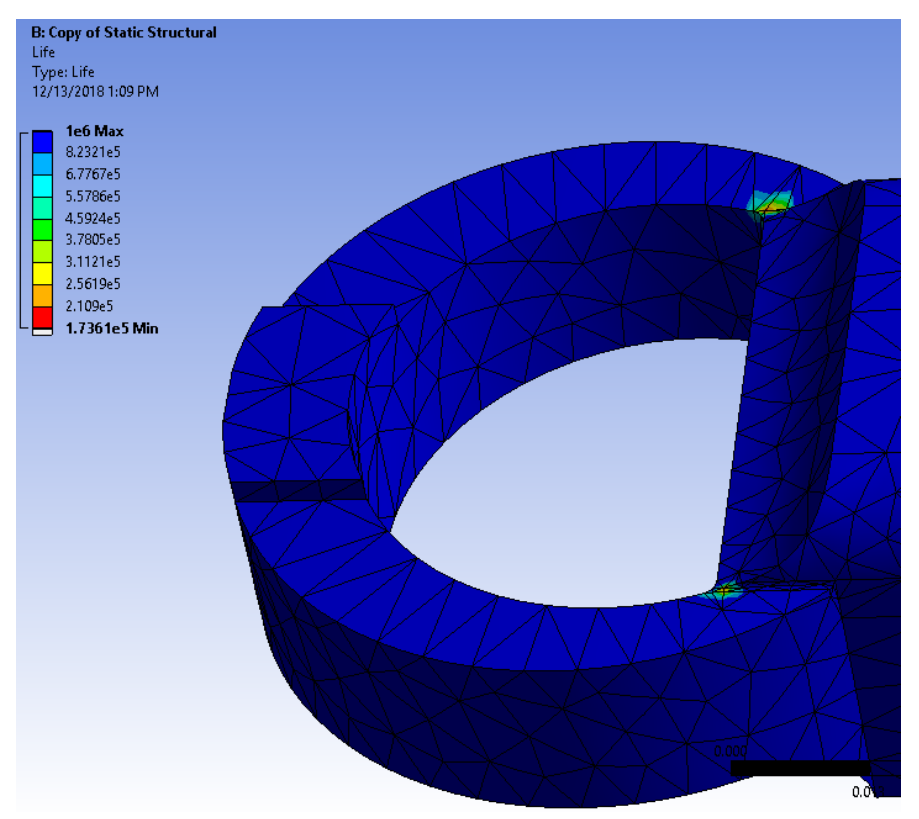

Figure 6: Fatigue life of Model

As shown in Figure 5, Model A fatigue life shows that the low life is concentrated on a single area, with a lifespan of roughly 107,000 cycles. In order to improve the fatigue life, the modification of adding a few cubic millimeters of material to the part is evaluated, as shown in the Model B in Figure 6.

In Figure 6, while the lower fatigue life area is spread out, but the life span is increased to roughly 174,000 cycles, an increase of nearly $70 \%$. This is because the small rounds added to model B, as no other settings were changed. With the rounds disrupting the stress concentration caused by edges, this result is quite reasonable. The design of improvement is simple, without causing a significant price increase, or requiring a large amount of analysis before the change is made.

\section{Course Outcome Assessment}

With the use of ANSYS for the course project, the course outcome has progressed positively. Figure 7 shows the course evaluation scores and the enrollment data in the recent three consecutive offering. 


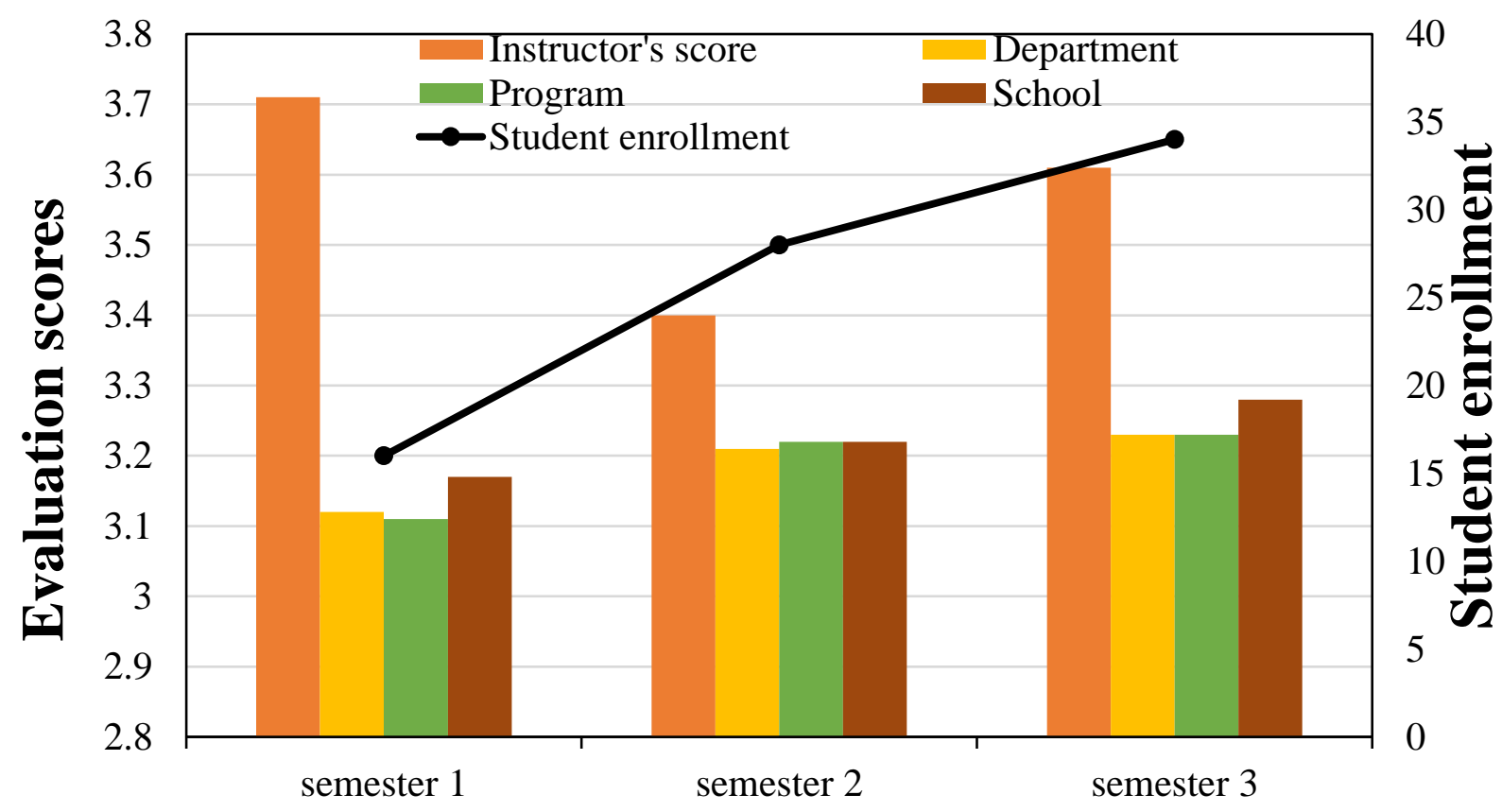

Figure 7: Course evaluation scores and student enrollment in the recent three semesters.

As shown in Figure 7, the instructor's course evaluation scores of the course are all substantially higher than $(>30 \%)$ the average scores of other courses offered in the department, program, and school, suggesting the popularity of the new revised course. The course evaluation scores do fluctuate over the three semesters, with semester 1 as the highest. This fluctuation is likely due to the different background of the students.

In terms of the number of student enrollment, the number of student has steady increased from 15 in semester 1 to 26 in semester 2, and 34 students in semester 3. The enrollment data show the increasing popularity of the course.

\section{Conclusion}

In this project, we developed a new project-based finite element modeling element in Mechanical Behavior of Materials class. Specifically, students are required to identify a project which requires to using a commercial finite element software package to simulate the mechanical response of the system. The major conclusions are summarized as follows:

1. The revised engineering course becomes increasingly popular with continued growth of enrollment.

2. The revised course contents are well received by the students, as illustrated by the high instructor's course evaluation scores over three semesters. 
3. Using fatigue analysis as a case study, it shows that FEA program is an effective learning tool to teach the traditional mechanics courses. Making simple changes for iterative design is simple and effective in FEA programs.

\section{References}

[1] B. Engel, Sara Salman, Hassan Al-Maeeni. Failure Analysis and Fatigue Life Estimation of a Shaft of a Rotary Draw Bending Machine. International Journal of Mechanical and Mechatronics Engineering. 2017; 11:1785-90.

[2] Samuel O Afolabi, Bankole I. Oladapo, Christianah O Ijagbemi, Adeyinka O.M. Adeoye, Joeph F. Kayode. Design and finite element analysis of a fatigue life prediction for safe and economical machine shaft. Journal of Materials Research and Technology. 2018 (https://doi.org/10.1016/j.jmrt.2017.10.007).

[3] Yu Ma, Yuwen Li. Fatigue life analysis of the van body frame based on ANSYS, $2^{\text {nd }}$ International Conference on Artificial Intelligence, Management Science and Electronic Commerce; 2011 Aug. 8-10; Dengleng, China.

[4] Sebastian Marian Zaharia. Fatigue life simulation of the specimens made of mechanical component, International Conference of Scientific Paper AFASES; 2015 May 28-30; Brasov, Romania

[5] Akash Swamy, B.S. Shenoy, I.N. Aparna. Effect of grid size on fatigue life calculations of a dental implant. Journal of Computational Methods in Sciences and Engineering 2017; 17:28993

[6] Kumar Vinod, Singh Gurdeep, Saxena Ravindra K. Investigation on fatigue life of RailWheel assembly using Finite Element Analysis, 2016 IEEE International Conference on Intelligent Rail Transportation (ICIRT). 2016 Aug 23-25. 